\title{
Decomposition of multi-mode signals using dispersion curves and Bayesian linear regression
}

\author{
Marcus Haywood-Alexander ${ }^{\mathrm{a}}$, Nikolaos Dervilis ${ }^{\mathrm{a}}$, Keith Worden ${ }^{\mathrm{a}}$, Gordon B. Dobie ${ }^{\mathrm{b}}$, and \\ Timothy J. Rogers ${ }^{\text {a }}$ \\ aDynamics Research Group, Department of Mechanical Engineering, Mappin Street, \\ University of Sheffield, Sheffield, S1 3JD, UK \\ ${ }^{\mathrm{b}}$ Electronic and Electrical Engineering, Royal College Building, 204 George Street, Glasgow, \\ G1 1XW, UK
}

\begin{abstract}
For certain structure types and damage sizes, guided waves offer some distinct advantages for damage detection, such as range and sizing potential, greater sensitivity and cost effectiveness. Guided waves exhibit multiple modes; for Lamb waves there are two types; symmetric and antisymmetric. In damage detection regimes, information and features of individual modes, which propagate from a single source, are useful for localisation and sizing of damage. This facet leads to the motivation to decompose a single signal into the individual modes that are received in the wave-packet. Decomposition of wave modes is possible in full-field Lamb wave data from a forward-backward, two-dimensional Fourier transform method that involves dispersion curve information; though this method cannot be applied directly to signals at a single location. By using this method, the expected nominal waves can be determined for a given propagation distance; i.e. the individual wave modes expected to be present regardless of damage. In the presence of damage, residual signals will be present which contain information on the damage. In this paper, a Bayesian linear regression technique is used to decompose single multi-mode signals into their individual wave modes, which is then used to determine any residual signals. This decomposition is made by determining the expected shape and size of individual mode signals from the full-field decomposed waves. The information inferred by this method, both before and after the wave has propagated through damage, is studied.
\end{abstract}

Keywords: guided wave, Lamb wave, mode decomposition, dispersion curves

\section{INTRODUCTION}

The use of ultrasonic guided waves (UGWs) for non-destructive evaluation (NDE) and structural health monitoring (SHM) strategies ${ }^{1}$ can offer a number of advantages, such as range and sizing potential, greater sensitivity and cost effectiveness. UGWs consist of two types of high-frequency stress waves: Rayleigh waves which propagate on a surface or, in plate-like media where the thickness is sufficiently small compared to the wavelength, as Lamb waves. An explanation of Rayleigh and Lamb waves is given by Viktorov ${ }^{2}{ }^{\text {Worden }}{ }^{3}$ and Rose,${ }^{4}$ though a short introduction will be given here. A particular characteristic of Lamb waves is their separation into symmetric and antisymmetric modes, the former of which has the upper and lower bounds oscillating in opposite directions at equal propagation distance, whereas the latter oscillates in the same directions. The number of each type of wave mode present increases with increasing frequency-thickness; these are termed higher-order modes. A wave-packet is a single burst containing multiple wave modes of different frequency and shape; for Lamb waves these will contain both types of modes. The propagation velocity of Lamb waves depends on the central frequency of the wave and will vary between the modes present, and therefore a wave-packet of mixed wavelengths will spread out in space, i.e. they will disperse.

The behaviour of these waves in the presence of damage varies between modes, therefore it is useful to be able to determine signal features that represent individual attributes of each mode present in a single wave-packet.

Further author information: (Send correspondence to M.H-A.)

M.H-A.: E-mail: mhaywood-alexander1@sheffield.ac.uk 
In the rest of this paper, the incident waves observed that are expected to be present regardless of damage are referred to as the nominal waves. A particular phenomenon that occurs when Lamb waves interact with damage is that of mode conversion,${ }^{5}$ where modes are converted into others as a result of variations in plate dimensions. This phenomenon is directly linked to reflection characteristics of damage, which is dependent on the size and shape of the inhomogeneity or damage. This characteristic interaction of Lamb waves with damage leads to the identification of features of a multi-mode wave-packet that offer insight into the location and/or severity of damage. Firstly, the amplitude ratio between the nominal antisymmetric and symmetric waves, will vary with damage as the reflection characteristics of each wave can be determined for damage size and shape. ${ }^{6,7}$ Where $A\left(A_{0}\right)$ and $A\left(S_{0}\right)$ are the amplitudes of the $A_{0}$ and $S_{0}$ mode respectively, this ratio is given by,

$$
R=\frac{A\left(A_{0}\right)}{A\left(S_{0}\right)}
$$

Furthermore, the energy in any converted/reflected modes will give information on the location because of the difference in attenuation and initial energy from the nominal wave modes. Another feature that will provide information on the location of damage will be the difference in the time of arrival between the nominal waves and any additional waves present; as any converted/scattered modes, despite having the same instantaneous group velocity, will have a different average velocity from the source. Although there has been evidence of useful techniques that do not require decomposition, via the use of piezoelectric rosettes, ${ }^{8}$ the cost of using such hardware may quickly rise for large-scale systems. A novel method has been proposed, involving the decomposition of threeway tensors constructed from 'actuator', 'sensor' and 'time dimensions' that shows robustness. ${ }^{9}$ However this method requires that data are collected from multiple actuation sources and sensor locations, so it may be advantageous in some systems to be able to localise using fewer such locations, thus reducing data usage and processing time.

In order to obtain the features discussed, it would be useful to derive the individual signals of all the waves present in a single multi-mode wave-packet signal, including any additional signals due to damage. Some multimode decomposition techniques have been proposed with a variety of approaches. One such approach is a parameter-based iterative procedure to match the shape of the wave with the physical equations governing a Lamb wave in homogeneous materials. ${ }^{10}$ Other methods involve the usage of a series of $1 \mathrm{D}$ and $2 \mathrm{D}$ bandpass filters on full-field laser scans over the area of wave propagation, ${ }^{11}$ identification of ratio features from signal processing of the received signal, ${ }^{12}$ and using concentric ring and circular PZTs. ${ }^{13}$

In this paper, a method is proposed which utilises prior knowledge of the nominal symmetric and antisymmetric modes to obtain information present in the signal as a result of the presence of damage. Lamb waves were simulated in a homogeneous plate using the local interaction simulation approach (LISA), ${ }^{14,15}$ for an undamaged plate and various damage scenarios. In order to obtain the individual nominal signals of a Lamb wave as it propagates through space, first a multi-mode separation technique was applied which utilises a forward-backward, two-dimensional Fourier transform (TDFT) method and dispersion curve information. ${ }^{16,17}$ These decomposed signals are then used to represent the expected nominal wave shape at a given location and are used as the basis for decomposing an individual signal into the nominal waves and residual waves. By using a Bayesian linear regression technique, it is shown here how a signal from a single location can be decomposed in order to determine the features outlined above.

Many current individual-signal decomposition methods require accurate previous models of guided wave propagation in the material being inspected, analytical models of which are difficult to attain accurately for complex materials. Such an example would be for fibre-matrix composites where the attenuation varies with respect to fibre-orientation. ${ }^{18}$ The method demonstrated here can be generalised further as a decomposition tool of waves in more complex materials because of its ability to be used with experimental datasets, either simulation based or from scanning laser tests.

\section{LAMB WAVE PROPAGATION IN PLATES}

Elastic waves in orthotropic, inhomogeneous media are described by the elastodynamic equation, ${ }^{19}$

$$
\partial_{l}\left(S_{k l m n} w_{m, n}\right)=\rho \ddot{w}_{k} \quad(k, l, m, n=1,3)
$$


where $S$ is the stiffness tensor, $\rho$ is the material density, $w$ is the displacement field in which the comma represents differentiation with respect to space and the double dot represents double differentiation with respect to time. In bounded media these waves will exhibit as Lamb waves, which in isotropic elastic media will exhibit in two distinct shapes: symmetric and antisymmetric. There are two characteristic equations for Lamb waves which describe their behaviour in given media:

$$
\frac{\tan (q d)}{\tan (p d)}=-\frac{4 k^{2} p q}{\left(q^{2}-k^{2}\right)^{2}}
$$

for the symmetric modes and,

$$
\frac{\tan (p q)}{\tan (q d)}=-\frac{4 k^{2} p q}{\left(q^{2}-k^{2}\right)^{2}}
$$

for the antisymmetric modes. The definitions of $p$ and $q$ are given by,

$$
p=\frac{\omega}{\sqrt{c_{L}^{2}-c^{2}}}, \quad q=\frac{\omega}{\sqrt{c_{T}^{2}-c}}
$$

where $k$ is the real wavenumber, $\omega$ is the central frequency, $c$ is the bulk wave speed, $c_{L}$ is the longitudinal wave speed and $c_{T}$ is the transverse wave speed. Given a value of $\omega$, eqs. (3) and (4) specify allowed values of $c$ for either mode; as $c$ is a function of $\omega$ this means that the waves are dispersive. Solutions to these equations are determined numerically, and plots of the wave velocity with respect to frequency-thickness are called dispersion curves.

\subsection{LISA Simulation}

The overall method of simulating guided waves using the local interaction simulation approach (LISA) is well documented ${ }^{14,15,20}$ but a brief overview will be given here. The LISA simulation method uses iterative difference equations and is based on a sharp interface model. This allows LISA to incorporate the effects of boundaries and inhomogeneities with ease - a primary benefit of using the method, as well as faster computing time in comparison to FEA. A key difference between this method and standard finite difference (FD) approaches is that the LISA solves a discrete form of eq. (2) exactly, modelling physical phenomena without other approximations, whereas the FD is the solution of the partial differential equation after discretisation.

A finite difference formulation is used on eq. (2) to generate iterative equations that can be applied for a given point in space. The derivations of these equations can be followed in, ${ }^{14}$ which is achieved by discretising the grid into evenly sized cells, and considering these cells as a series of springs and masses which populate the medium. At each point, which is neighboured by 8 cells, the sharp-interface model is used to average the properties of the neighbouring cells. It is assumed at a point that the material properties and displacements are continuous, whereas interfaces of cells are treated as discontinuous. As the final iterative equations are quite lengthy and not the focus of this paper, they are not given here, but the reader can refer to the work by Delsanto et al. ${ }^{14}$

\begin{tabular}{|l|l||l|l|}
\hline Material & Aluminium & Density, $\rho$ & $2710 \mathrm{~kg} / \mathrm{m}^{3}$ \\
\hline Longitudinal sound speed, $c_{L}$ & $6420 \mathrm{~m} / \mathrm{s}$ & Transverse sound speed, $c_{T}$ & $3040 \mathrm{~m} / \mathrm{s}$ \\
\hline Width x Length & $300 \times 300 \mathrm{~mm}$ & Plate thickness & $1 \mathrm{~mm}$ \\
\hline Cell dimension, $\varepsilon$ & $0.25 \mathrm{~mm}$ & Time step, $\tau$ & $0.01496 \mu \mathrm{s}$ \\
\hline
\end{tabular}

Table 1: Properties of Lamb wave in plate simulation performed using LISA approach.

Using the LISA approach here, Lamb waves were simulated in an aluminium plate, the details of which can be found in Table 1. First a wave was simulated in an undamaged plate, then the simulation was repeated with a $1 \mathrm{~mm}$ square, half-thickness notch at $x, y=75,150 \mathrm{~mm}$. For all simulations, the actuation source is a $20 \mathrm{~mm}$ diameter piezo-electric transducer placed at the centre of the plate, with a $1 \mathrm{MHz}$, five-cycle, Hanning windowed sine wave. This source is represented using a linear systems model. ${ }^{15}$ The results of the damaged and undamaged plate simulations can be seen in Figure 1.

Figure 1 shows how the LISA simulation captures the effect of damage on the wave propagation well, as the reflections are clearly seen of the A0 mode. It can be also be seen how the boundaries reflect the waves, this is an important consideration in the method as this can have effects on the technique, this will be discussed later on. 


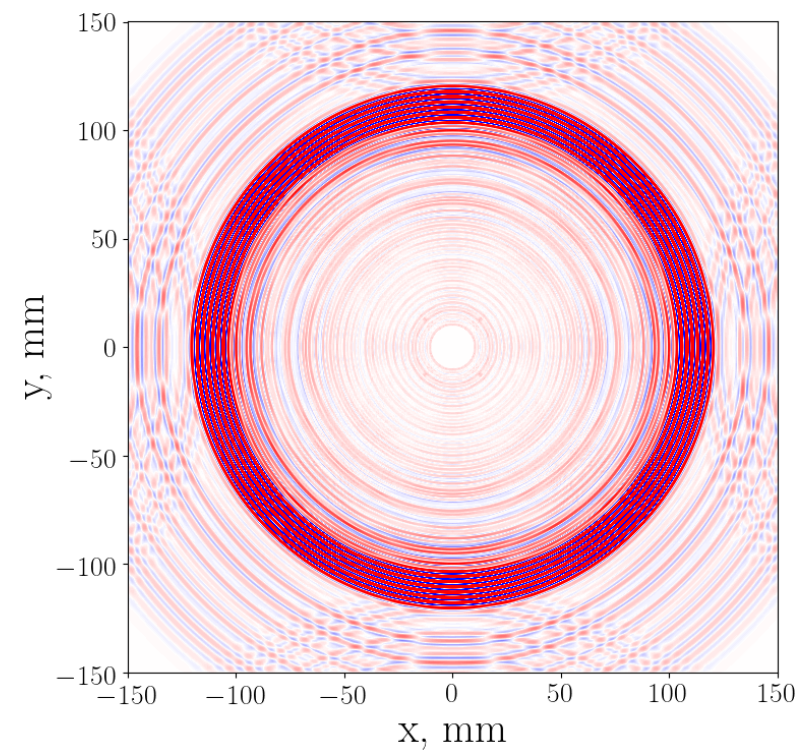

(a)

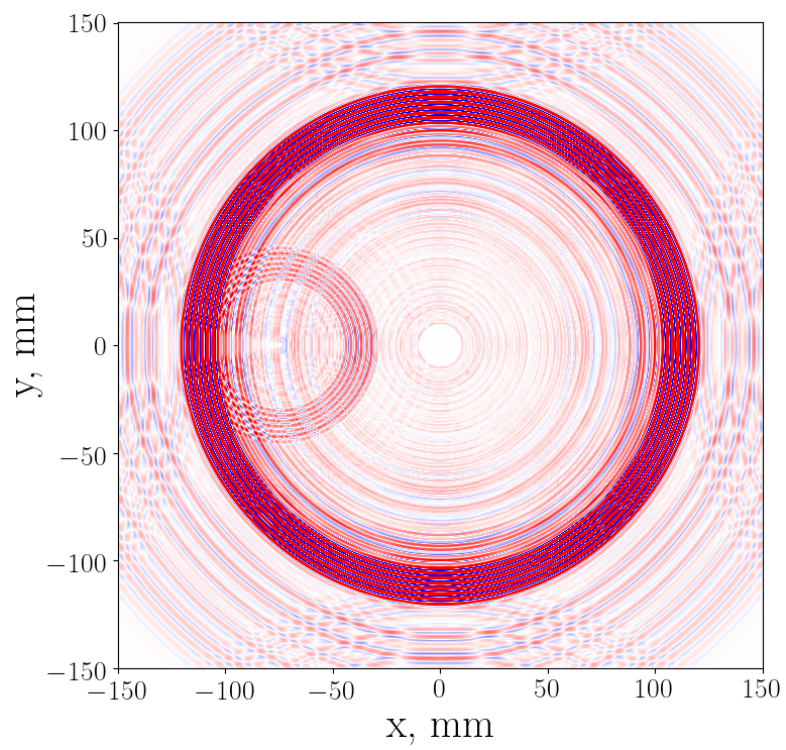

(b)

Figure 1: Surface displacement results of LISA simulation for (a) an undamaged plate and (c) a damaged plate at $35 \mu \mathrm{s}$. Dimensions are altered to place actuation source at the origin.

\section{SEPARATION OF MULTI-MODE SIGNALS}

As discussed, it would be advantageous to decompose an individual multi-mode Lamb wave signal into individual modes in order to obtain features that are useful for determining the severity and location of damage. The following section will explain the multi-stage process of the decomposition technique developed which utilised an initial full-field based method to establish the expected shape of individual modes, and then using a model learning technique to decompose an individual signal of the damaged case into the nominal modes and residual waves.

\subsection{Full-field multi-mode separation}

Separation of multi-mode signals of a Lamb wave over its propagation in space is carried out by a forwardbackward method using the experimentally determined dispersion curve. The method is explained in detail by Haywood-Alexander et al. ${ }^{17}$ but a brief explanation is provided here. First the dispersion curve must be obtained by spatially sampling the surface displacement of a Lamb wave as it propagates through a bounded medium, then one performs a two-dimensional Fourier transform (TDFT) along time and distance sequentially. ${ }^{16}$ The spatial sampling was done here by extracting the simulation surface displacement data at all points where $x<150$ and $y=0$. This strategy gives a transformation from the time-distance $[t-x]$, space to the frequency-wavenumber $[f-k]$, space,

$$
H(f, k)=\int_{-\infty}^{\infty} \int_{-\infty}^{\infty} h(t, x) e^{-i 2 \pi(f t+k x)} d t d x
$$

where $h(t, x)$ is the surface displacement with respect to time $t$ and distance $x$. The resulting image data, which shows dispersion curve information, are shown in Figure 2.

Then, a simple ridge-picking algorithm is used to extract local maxima in the image data $H$, and generates a Boolean image mask data $D$ of the same size as $H$. A buffer of $\pm u$ and $\pm v$ additional data points, in the horizontal and vertical directions respectively, are set to be 1 in $D$, in order to reduce information loss from the $[f-k]$ space. D can also be tuned to only include curves representing selected wave modes to reconstruct. The 'flattened' $[f-k]$ data is then determined by,

$$
H_{*}=D \circ H
$$




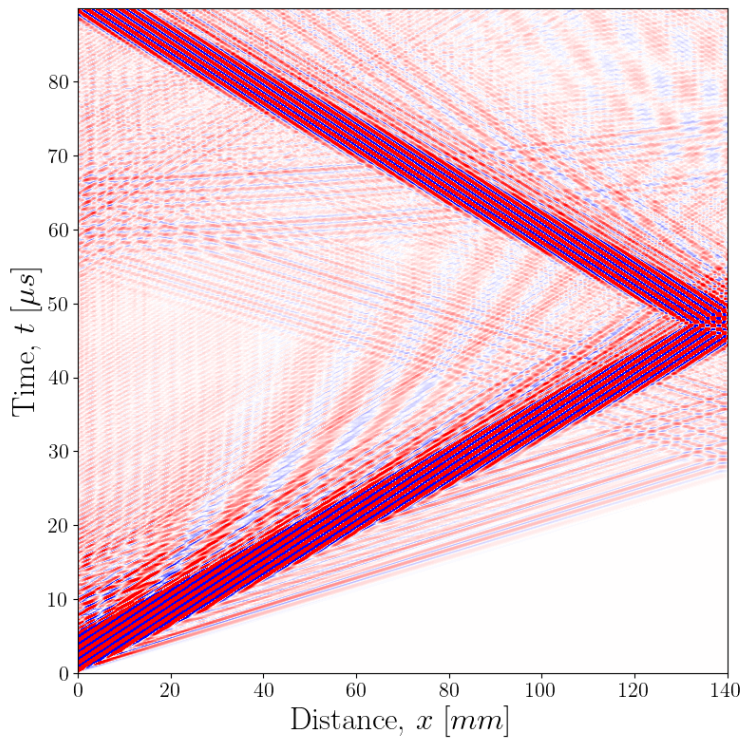

(a)

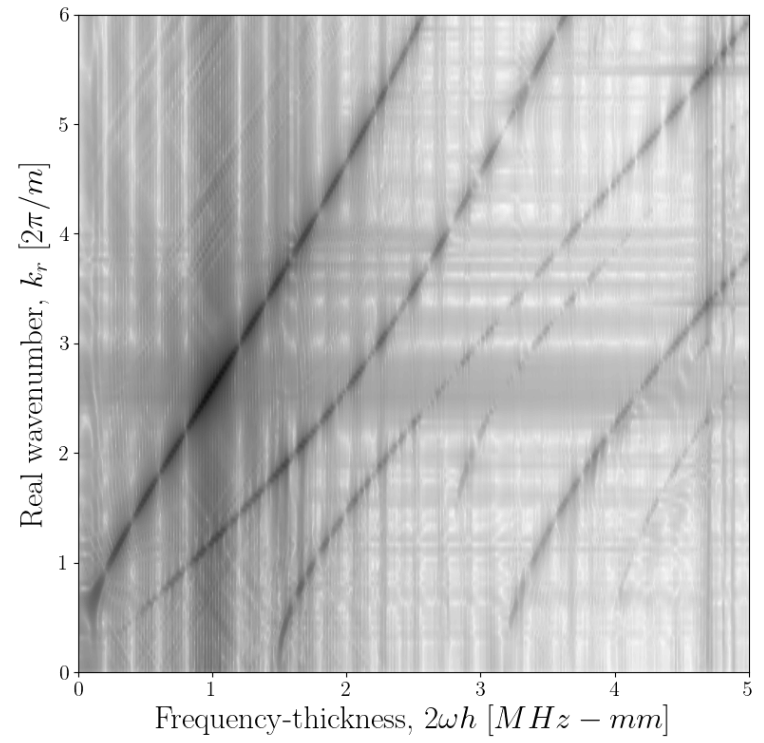

(b)

Figure 2: Example of dispersion information determined from a TDFT by extracting an example of the $[t-x]$ data from the LISA simulation. Shown in terms of (a) $h(t, x)$ and (b) $H(f, k)$

Once these curves are extracted, they can be used to reconstruct individual, or selected, modes by applying an inverse two-dimensional Fourier transform (ITDFT) on the new image data,

$$
h_{*}(t, x)=\int_{-\infty}^{\infty} \int_{-\infty}^{\infty} H_{*}(f, k) e^{i 2 \pi(f t+k x)} d f d k
$$

This method requires normalisation of the signal at each distance point, though it is trivial to return the range of the decomposed wave to that of the measured signal by storing the normalisation parameter. For this work it is kept at its normalised values as the decomposition method is focussed on just the shape of the expected nominal signals and not their amplitude. Advantages and caveats of this method are discussed by Haywood-Alexander et al. ${ }^{17}$

\subsection{Individual multi-mode separation}

Once the expected nominal waves were determined, they were used to decompose an individual signal into the nominal residual signals. This was done using a Bayesian linear regression technique, a detailed description can be found in the text by Murphy. ${ }^{21}$

Traditional linear regression formulates a model using point estimates of a set of parameters which "best" fit an available dataset, based on minimising an $L^{2}$-norm between the model predictions and the data. Instead, BLR aims to establish a probability distribution of possible model parameters. The model has the form,

$$
y=\mathbf{w}^{\top} \phi(\mathbf{x})+\varepsilon, \quad \varepsilon \sim \mathcal{N}\left(0, \sigma^{2}\right)
$$

where $\phi$ is some basis for expansion of a $p$-dimensional data point $\mathbf{x} ; \mathbf{w}=\left\{w_{1}, w_{2}, \ldots, w_{p}\right\}$ are the associated weights of the basis expansion, and $\varepsilon$ is an additive Gaussian white noise distributed as $\mathcal{N}\left(0, \sigma^{2}\right)$. The weights $\mathbf{w}$ and the variance $\sigma^{2}$ are the unknowns. The Bayesian linear regression model approach was chosen since it returns a quantified uncertainty. The task is then to compute the posterior distribution of the parameters $p\left(\mathbf{w}, \sigma^{2} \mid D\right)$. This posterior distribution has the form,

$$
p\left(\mathbf{w}, \sigma^{2} \mid \mathcal{D}\right)=N I G\left(\mathbf{w}, \sigma^{2} \mid \mathbf{w}_{N}, \mathbf{V}_{N}, a_{N}, b_{N}\right)
$$




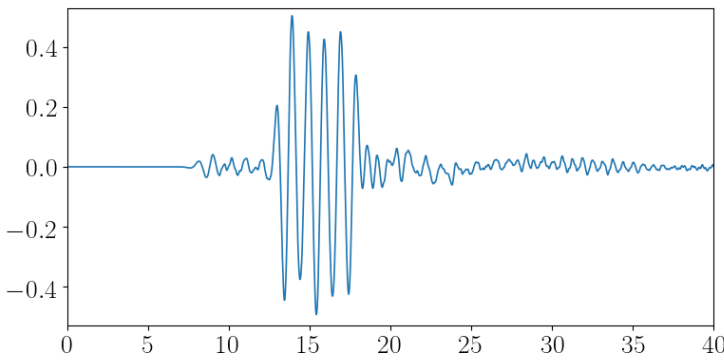

(a)

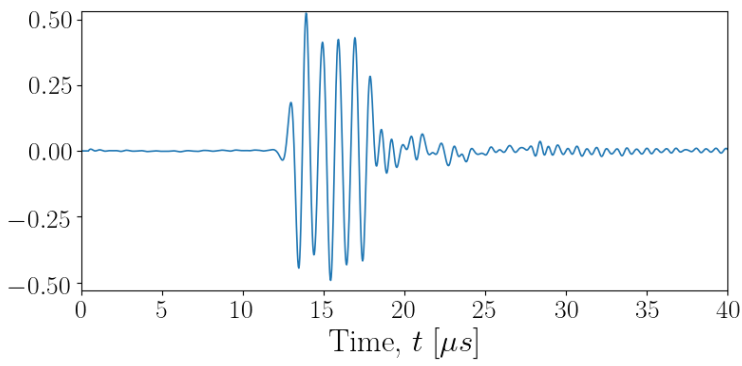

(c)

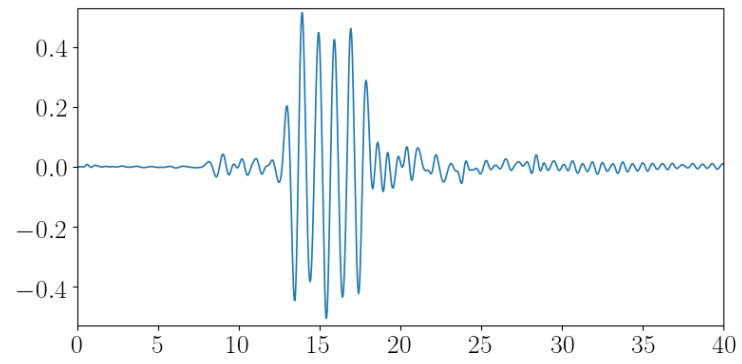

(b)

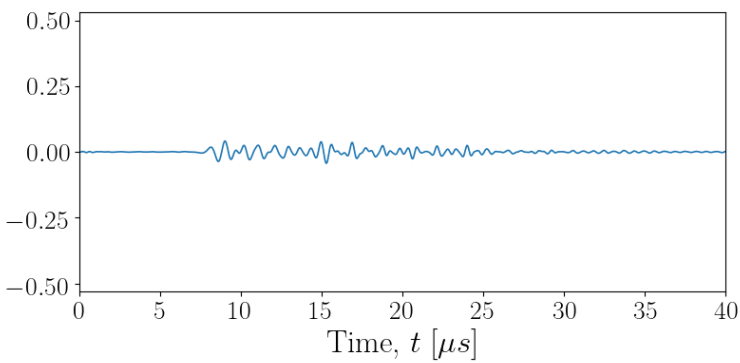

(d)

Figure 3: Individual signals from the full-field TDFT multimode decomposition for a $1 \mathrm{MHz}, 5$ cycle sine wave at a propagation distance of $50 \mathrm{~mm}$ for (a) original multimode signal, (b) reconstructed sum of A0 and S0 modes, (c) reconstructed A0 mode and (d) reconstructed S0 mode.

with,

$$
\begin{gathered}
\mathbf{w}_{N}=\mathbf{V}_{N}\left(\mathbf{V}_{0}^{-1} \mathbf{w}_{0}+\mathbf{X}^{\top} \mathbf{y}\right) \\
\mathbf{V}_{N}=\left(\mathbf{V}_{0}^{-1}+\mathbf{X}^{\top} \mathbf{X}\right)^{-1} \\
a_{N}=a_{0}+n / 2 \\
b_{N}=b_{0}+\frac{1}{2}\left(\mathbf{w}_{0}^{\top} \mathbf{V}_{0}^{-1} \mathbf{w}_{0}+\mathbf{y}^{\top} \mathbf{y}-\mathbf{w}_{N}^{\top} \mathbf{V}_{N}^{-1} \mathbf{w}_{N}\right)
\end{gathered}
$$

where $\mathbf{V}_{0}, \mathbf{w}_{0}, a_{0}$ and $b_{0}$ are hyperparameters of the prior. It is possible to set an less informative prior for $\sigma^{2}$ by applying $a_{0}=b_{0}=0$. Also setting $\mathbf{w}_{0}=0$ and $\mathbf{V}_{0}=g\left(\mathbf{X}^{\top} \mathbf{X}\right)^{-1}$ for any positive value $g$; leads to Zellner's g-prior. ${ }^{22}$ By having the prior variance proportional to $\left(\mathbf{X}^{\top} \mathbf{X}\right)^{-1}$, it is ensured that the posterior is invariant under scaling of the inputs.

For this work, the input data $\mathbf{x}=\left\{x_{A 0}, x_{S 0}\right\}$, where $x_{i}$ is a column vector of the normalised nominal wave for mode $i$, the target vector $\mathbf{y}$ is the measured signal. The nominal waves are also re-normalised to their individual ranges in order to better compare the predicted weights between the wave modes. One could consider $\phi$ to be the decomposition of the expected nominal signal into the selected modes. For this work, only the fundamental modes are used, although it would be trivial to use the same method on more selected modes. For this method, any signal range can be used, though it is preferable to use a normalised signal. The expected weight parameters $\mathbf{w}$ will give relative amplitudes of the nominal waves, directly influenced by the range of $\mathbf{x}$. For computational reasons, it is preferable to use the normalised ranges for these wave vectors as any simultaneous loss in amplitude of the nominal waves will not be captured.

An important metric that is attainable from the Bayesian linear regression method is the predictive likelihood, which gives an indication of the likelihood that the model fits and takes into account the uncertainty as well as the quality of the mean fit. As this method uses a tractable Gaussian posterior, the predictive likelihood is given by,

$$
p(\tilde{\mathbf{y}} \mid \tilde{\mathbf{x}}, \mathbf{y}, \mathbf{x})=\mathcal{N}(\mathbb{E}[\tilde{\mathbf{y}}], \mathbb{V}[\tilde{\mathbf{y}}])
$$


where $\mathbb{E}[\tilde{\mathbf{y}}]$ and $\mathbb{V}[\tilde{\mathbf{y}}]$ are the predicted values and variance of the output given input $\mathbf{x}$. For computational stability this likelihood is calculated in the log space, and is named the independent predictive log-likelihood $P L L_{i}$ and is defined by,

$$
P L L_{i}=\sum_{i}^{N} \log \mathcal{N}\left(\mathbf{y}_{i} \mid \mathbb{E}\left[\mathbf{y}_{i}\right], \mathbb{V}\left[\mathbf{y}_{i}\right], \mathbf{w}\right)
$$

for $N$ data points. This value is the product over the predictive likelihoods for every point, i.e. the joint likelihood if they were uncorrelated.

\section{RESULTS}

Some examples of decomposed signals from the damaged scenario are shown in Figure 4. These are taken at selected distances along the propagation path directly between the actuator and the damage. The figures show the measured surface displacement at each location and the expected surface displacement because of the nominal waves, predicted using the measured displacement. Below this, the figure shows the residual of these two signals, giving information on any extra waves that propagate through this location because of the damage. The figures indicate the method provides a good estimate of the decomposed modes from the individual signal.
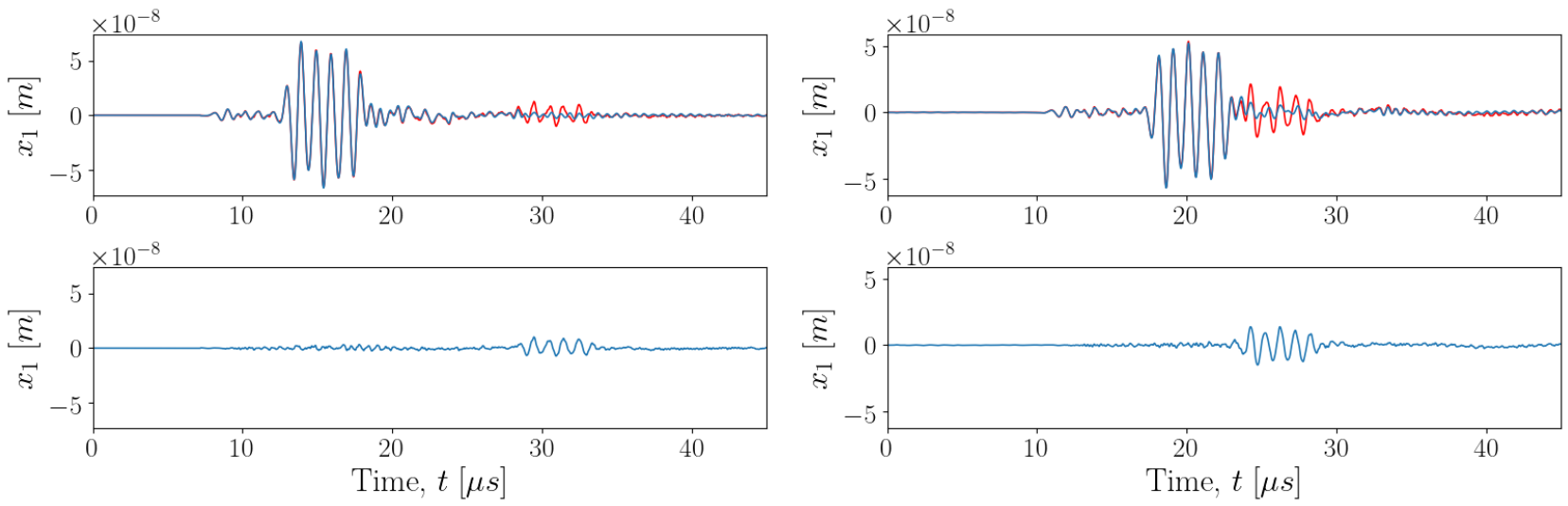

(a)

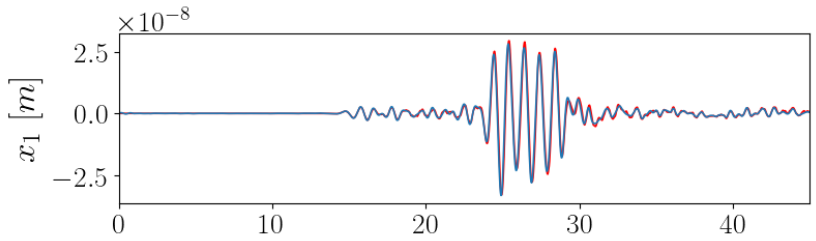

(b)
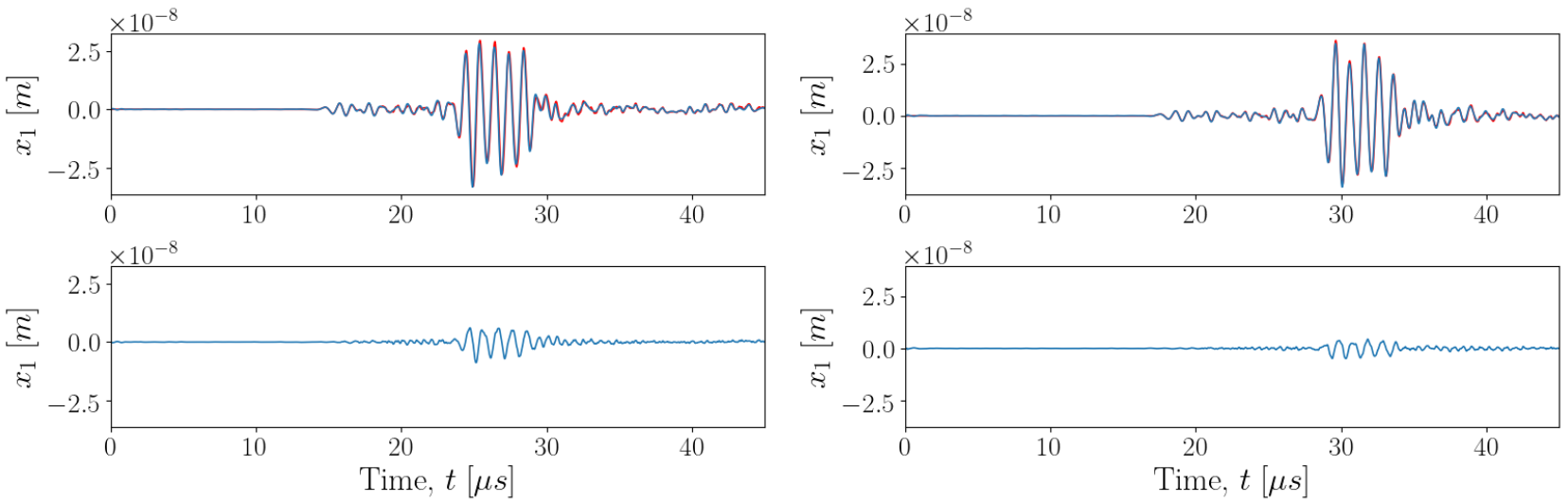

(c)

(d)

Figure 4: Decomposed multi-mode signals of damaged plate at (a) $50 \mathrm{~mm}$, (b) $65 \mathrm{~mm}$, (c) $85 \mathrm{~mm}$ and (d) $100 \mathrm{~mm}$ from the actuation source. For each propagation distance, the top figure shows the measured signal in red and the predicted nominal waves signal in blue, the bottom figure shows the residual between the measured and predicted nominal. 
From Figures $4 \mathrm{a}$ and $4 \mathrm{~b}$, the most prevalent change of signals from the damage, at propagation distances before reaching damage, is the additional waves present due to reflection from the damage. The reflected S0 and A0 waves can be seen in Figure 4a at $16 \leq t \leq 20 \mu s$ and $28 \leq t \leq 34 \mu s$ respectively. These reflections are more spread out in time in Figure 4a than in Figure 4b, which is expected as a result of the higher group velocity of $\mathrm{S} 0$ than A0. In Figures $4 \mathrm{c}$ and $4 \mathrm{~d}$, there appears to be information in the residual signal, which has a similar shape to that of the antisymmetric mode. This similarity is likely the result of the error between the predicted and the measured wave, although it should be noted that this error is higher in comparison to any error present in the prediction of the S0 mode, and any errors in prediction of both modes in the signals before damage.

Figure 5 shows a closer look at the expected nominal waves as well as the predicted variance of this signal, taken at a propagation distance of $50 \mathrm{~mm}$, i.e. $25 \mathrm{~mm}$ away from the damage. For most of the time series, the measured signal falls within the bounds given by the predicted variance. An obvious section in which it does not fit within the variance bounds is for the reflection signals at $27 \leq t \leq 35 \mu \mathrm{s}$; however, the measured signal does also appear to exceed this boundary at $9 \leq t \leq 12 \mu \mathrm{s}$ and $17 \leq t \leq 26 \mu \mathrm{s}$. These areas correspond to the later section of the incident S0 mode and the signal following the arrival of the incident A0 mode respectively.

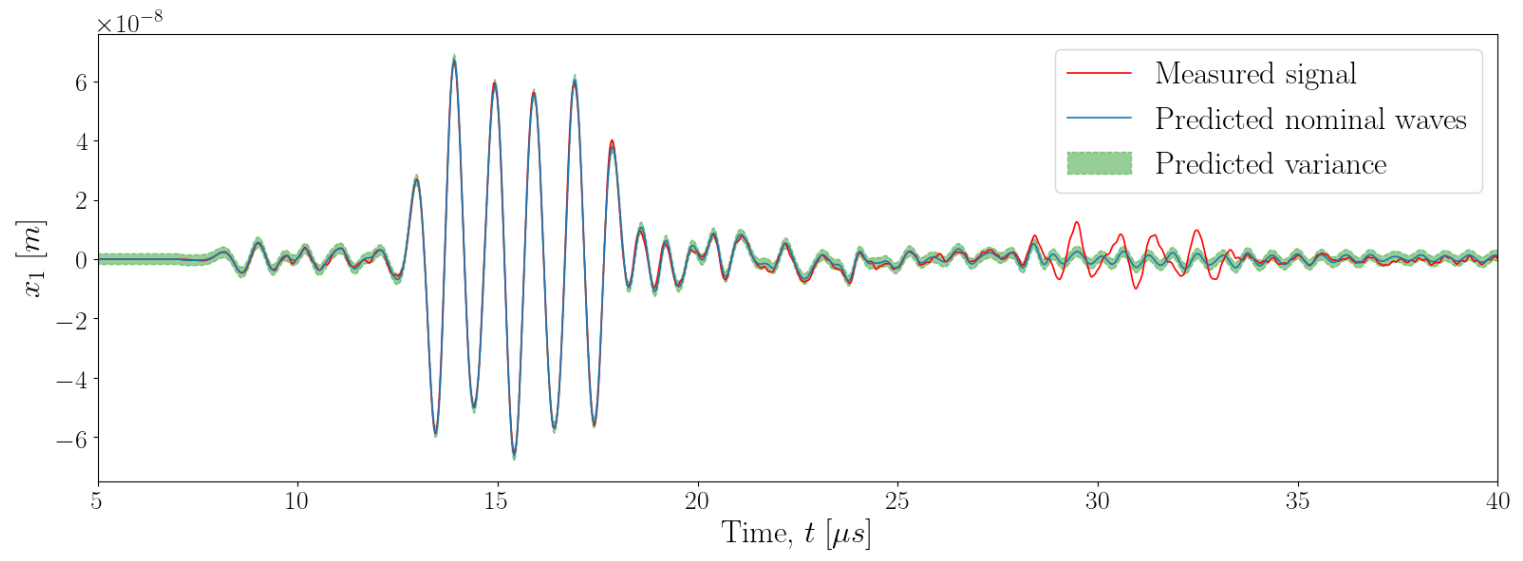

Figure 5: Measured signal and predicted nominal signal at a propagation distance of $50 \mathrm{~mm}$, with the predicted variance of the nominal wave signal shown in green bounds.

The decomposition method was applied at various propagation distances in the direction of the damage and the metrics inferred from the BLR method at each of these distances are shown in Figure 6. Firstly, the calculated weights are shown in Figure 6a, where the weights are written as the corresponding weight for each mode, $w_{A 0}$ and $w_{S 0}$. These weights can be interpreted as parameters relating the shape of the expected nominal wave to the range of the measured nominal wave. It is clear to see the attenuation of each mode as the propagation distance increases, although an interesting point to note is the sharp drop in the antisymmetric parameter $w_{A 0}$, after the wave has propagated through damage. There is a periodic nature to the weights with respect to the propagation distance, this is likely to be because of reflected waves coming in and out of phase with the nominal waves. The superposition of these waves will affect the amplitude of the nominal waves as the phase differences between the waves change.

The predicted variance of the predicted nominal waves at varying propagation distances is shown in Figure 6b; as the propagation distance approaches damage, the predicted variance increases smoothly. After the wave has propagated through the damage, there is a sharp drop in the predicted variance, although it is important to note this variance is not based on normalised signal data and is therefore directly influenced by the amplitude of the wave.

Figure $6 \mathrm{c}$ shows the predictive log-likelihood metric at each propagation distance. There does not appear to be any discernible pattern in this metric with respect to the location of damage, although there is a periodic pattern that could also be attributed to overlapping waves coming in and out of phase. This may indicate that the overlapping waves are not just reflected waves, but wave modes with different group velocities originating from the same source. Furthermore, the likelihood appears more irregular after a propagation distance of approx. 


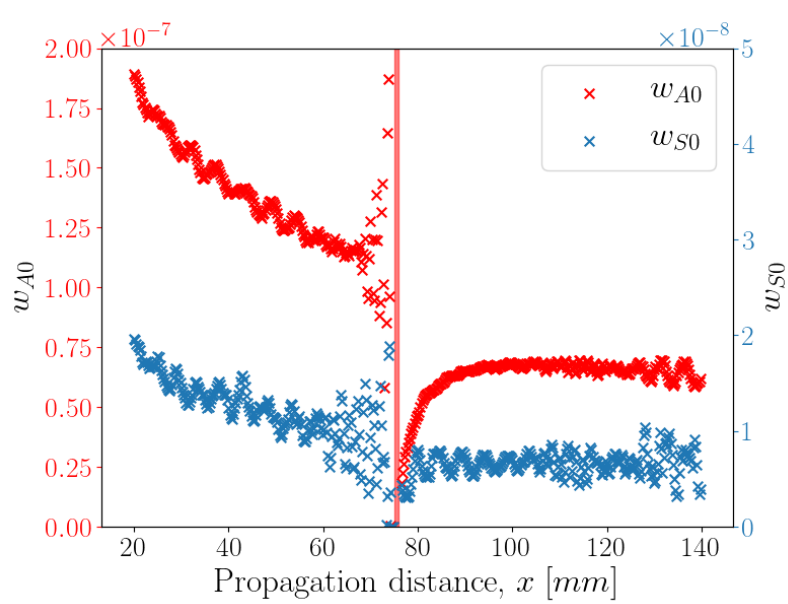

(a)

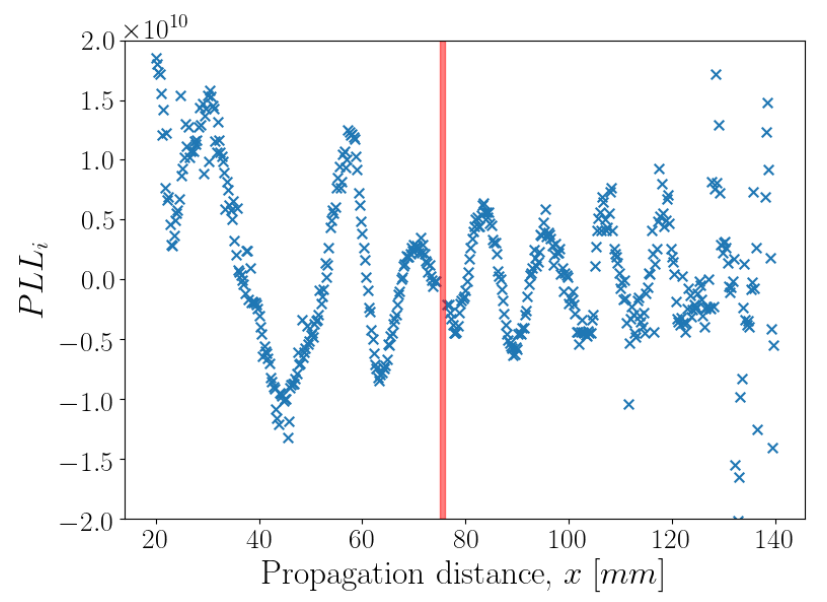

(c)

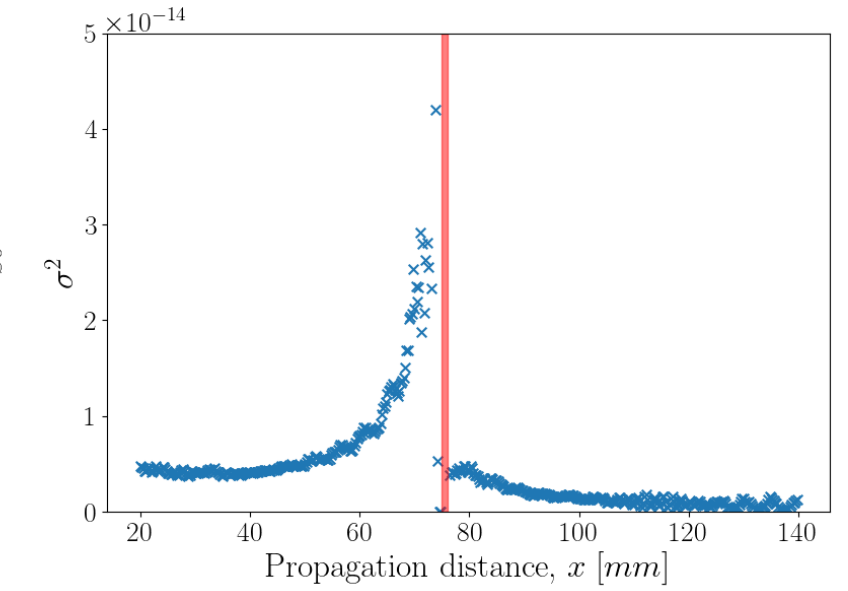

(b)

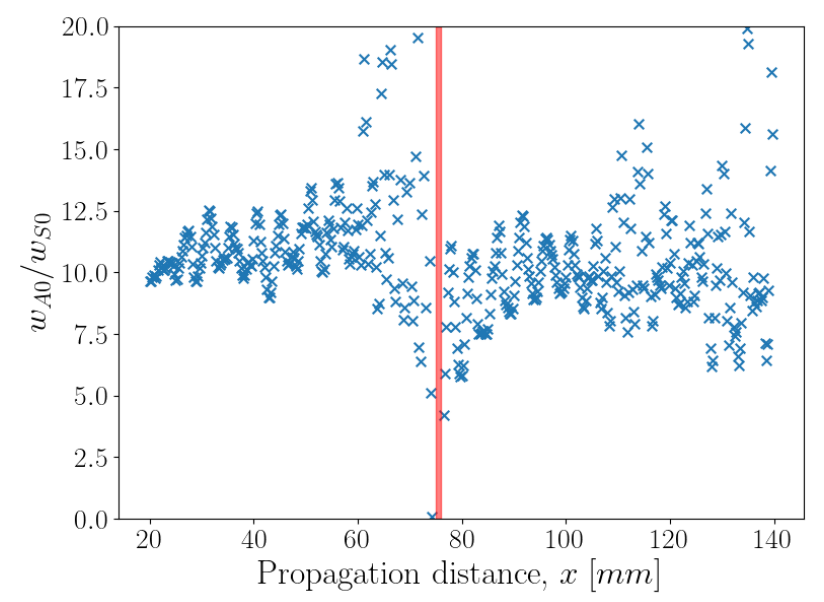

(d)

Figure 6: Metrics extracted from the BLR decomposition method at various propagation distances; (a) corresponding weights of each mode, (b) predicted variance of the nominal signal, (c) independent predictive loglikelihood and (d) ratio of A0 to S0 mode, calculated as the ratio of the calculated weights. For all figures, the area indicating damage is given by the filled red area.

$120 \mathrm{~mm}$; this is likely to be a result of the reflections from the boundary causing a much more complex multi-mode signal.

Finally, the ratio of the A0 mode to the S0 mode is shown in Figure 6d presented as the ratio of the corresponding weights. There is once again a periodic nature similar to that in Figure 6a, likely attributed to reflected/overtaking waves coming in and out of phase with each other. Much like the $P L L_{i}$ metric, as propagation distance approaches the location of damage, this metric becomes more irregular; a similar behaviour can also be seen for each individual weight in Figure 6a.

\section{DISCUSSION}

The predicted nominal waves shown in Figure 4 indicates the capability of the proposed method for decomposition of individual multi-mode signals. By decomposing multi-mode signals, it is easier to automatically determine features of individual modes that can be attributed to damage characteristics such as size and location. In most Lamb wave studies, in order to attain signals of individual modes, the structures are excited with tuned actuation (both in frequency and mode shape). Although this works well, it limits the available upper frequency 
limit of actuation, particularly in experimental regimes. Furthermore, the data recording/processing times are multiplied by the number of modes investigated using this tuned actuation technique. The residuals between the predicted nominal waves and measured signals show information that only exists due to damage. It can be seen in the residuals that there is a clear difference in the time of arrival of the reflected S0 and A0 modes, providing more dimensions to a feature set that can be used to localise damage.

The residuals shown in Figures $4 \mathrm{c}$ and $4 \mathrm{~d}$ show that, when decomposing the signal after it has been propagated through damage, there is a greater error in prediction of the A0 mode, in comparison to predictions made before the wave has been propagated through damage. This could be because of energy decreases in the A0 mode as a result of damage (as indicated by Figure 6a), as also there does not appear to be as much increase in error of prediction of the S0 mode. Mode conversion phenomena does not appear to have been evident in this simulation and is likely attributed to the reflection characteristics resulting from the relationship between wavelength and notch size with reflection coefficients. ${ }^{6,7}$ Therefore, this may indicate that there is more information in residual signals at locations which capture reflection/scattering of waves due to damage, rather than mode conversion.

In Figure 5, it can be seen more clearly when the signal deviates from the predicted signal given no presence of damage, and the regions where the measured signal exceeds the predicted variance boundaries have been pointed out. The strongest deviation is that of the reflection at $27 \leq t \leq 35 \mu \mathrm{s}$, which far exceeds the boundary. The deviation at $9 \leq t \leq 12 \mu s$ is likely attributed to reflections of the S0 mode from the damage, and at $17 \leq t \leq 26 \mu \mathrm{s}$ is likely from additional higher-order modes present. In this case, as the deviations due to reflection are much stronger than the others, it is suggested to rely on information given from this section of the signal.

The behaviour of the weights determined for each signal follows a trend that would be expected from the attenuation of the waves in the material, indicating that these could be used as energy-based features directly. The sharp drop, and subsequent steep rise, in the weight corresponding to the A0 mode may give insight to the behaviour of this mode as it propagates through damage. As the damage is a notch beginning on the same surface on which the displacement is measured and actuation occurs, the wave must pass under this when propagating through. The steep rise could be attributed to the energy of the wave diffusing from the lower portion of the plate thickness back to the upper surface after passing through the damage. The periodicity attributed to the phase difference of passing waves is an important aspect to note of this method; energy features based from this must incorporate this variance if used for any outlier detection strategies.

Furthermore, the ratio of the A0 mode to the S0 follows a trend that is expected in the presence of damage; the ratio drops after damage as the energy of the antisymmetric mode is more strongly affected. The exact nature of the trends of the energy features will depend on the wavelength and damage size, as shown by Lowe et al. ${ }^{6,7}$ The periodicity is once again exhibited in the ratio metric, supporting the idea that this is a result of phase difference between passing waves. As the S0 and A0 waves have different velocities, they will be in phase with passing waves at different points.

At propagation distances close to the damage and system boundary, the energy-based features begin to deviate from the expected attenuation trend, as well as the predictive likelihood when close to the plate boundary. This irregularity indicates that the method is not as successful at locations close to either damage or plate boundaries, i.e. at locations where reflections interfere strongly with the nominal waves. The increase in predicted variance close to damage further reinforces this, indicating that interference from reflections due to the damage cause a larger variance in the predicted nominal signals.

An advantage of this method is how computationally efficient each stage is, with the highest computational expenses being during the full-field mode separation stage, after which the nominal wave data can be stored for quick access. This asset leads to the benefit of greater applicability in real-time sensing scenarios, where speed of signal processing for damage detection is preferable. Furthermore, this method only requires data on the surface displacement from the actuation source as it propagates in one direction. This allows the method to be used for scenarios where accurate analytical modelling is costly or not possible, such as complex materials or geometries.

\section{CONCLUSION}

In this paper, a method has been proposed to decompose individual multi-mode signals into selected modes, based on a linear regression model which maps nominal signals whilst simultaneously capturing the variance. The 
method shows promise with some advantages over other methods, such as its readiness for use on experimental data, simple calculation methods and inherent feature-indicative metrics. The method has been applied to a damaged plate, showing how it can be used to extract signals that contain information due to the presence of damage. Parameters from the method have been analysed to determine its characteristics when used at varying propagation distances; these showed that consideration of the sensing location with respect to the damage and boundaries must be taken. Further work will be done on phase/distance alignment in order to utilise this method on cross-modelling strategies, such as laser scanning for the nominal waves and PZT sensing of the individual signal.

\section{ACKNOWLEDGMENTS}

The authors gratefully acknowledge the support of the UK Engineering and Physical Sciences Research Council (EPSRC) [grant numbers EP/R004900/1 and EP/R003645/1].

\section{REFERENCES}

[1] Rose, J., "Ultrasonic guided waves in structural health monitoring," Key Engineering Materials 270-273, 14-21 (2004).

[2] Viktorov, I., [Rayleigh and Lamb Waves: Physical Theory and Applications], Plenum Press (1967).

[3] Worden, K., "Rayleigh and Lamb Waves - Basic Principles," Strain 37, 167-172 (2001).

[4] Rose, J., [Ultrasonic waves in solid media], Cambridge University Press (2014).

[5] Alleyne, D. N. and Cawley, P., "The interaction of Lamb waves with defects," IEE Transactions on Ultrasonics, Ferroelectrics, and Frequency Control 39, 381-397 (1992).

[6] Lowe, M. J. S. and Diligent, O., "Low frequency reflection characteristics of the s0 Lamb wave from a rectangular notch in a plate," The Journal of the Acoustical Society of America 111, 64-74 (2002).

[7] Lowe, M. J. S., Cawley, P., Kao, J.-Y., and Diligent, O., "The low frequency reflection characteristics of the fundamental antisymmetric Lamb wave a0 from a rectangular notch in a plate," The Journal of the Acoustical Society of America 112, 2612-2622 (2002).

[8] Kijanka, P., Manohar, A., Lanza di Scalea, F., and Staszewski, W., "Damage location by ultrasonic Lamb waves and piezoelectric rosettes," Journal of Intelligent Material Systems and Structures 26, 1477-1490 (2015).

[9] Rébillat, M. and Mechbal, N., "Damage localization in geometrically complex aeronautic structures using canonical polyadic decomposition of Lamb wave difference signal tensors," Structural Health Monitoring 19, 305-321 (2020).

[10] Zhou, K., Zheng, Y., Zhang, J., Xu, X., Ma, S., and Wu, Z., "A reconstruction-based mode separation method of Lamb wave for damage detection in plate structures," Smart Materials and Structures 28, 035033 (2019).

[11] Tian, Z. and Yu, L., "Lamb wave frequency-wavenumber analysis and decomposition," Journal of Intelligent Material Systems and Structures 25, 1107-1123 (2014).

[12] Park, I., Jun, Y., and Lee, U., "Lamb wave mode decomposition for structural health monitoring," Wave Motion 51, 335-347 (2014).

[13] Yeum, C. M., Sohn, H., and Ihn, J. B., "Lamb wave mode decomposition using concentric ring and circular piezoelectric transducers," Wave motion 48, 358-370 (2011).

[14] Delsanto, P. P., Schechter, R. S., and Mignogna, R. B., "Connection machine simulation of ultrasonic wave propagation in materials iii: The three-dimensional case," Wave Motion 26, 329-339 (1997).

[15] Dobie, G., Spencer, A., Burnham, K., Pierce, S. G., Worden, K., Galbraith, W., and Hayward, G., "Simulation of ultrasonic Lamb wave generation, propagation and detection for a reconfigurable air coupled scanner," Ultrasonics 51(3), 258-269 (2011).

[16] Alleyne, D. and Cawley, P., "A two-dimensional Fourier transform method for the measurement of propagating multimode signals," The Journal of the Acoustical society of America 89, 1159-1168 (1991). 
[17] Haywood-Alexander, M., Worden, K., Dobie, G., Rogers, T. J., and Dervilis, N., "Lamb wave mode separation using dispersion curves," Proceedings of the 8th International Conference on Uncertainty in Structural Dynamics (2020).

[18] Haywood-Alexander, M., Dervilis, N., Worden, K., Cross, E. J., Mills, R. S., and Rogers, T. J., "Structured machine learning tools for modelling characteristics of guided waves," Mechanical Systems and Signal Processing 156, 107628 (2021).

[19] Achenbach, J. D., [Wave Propagation in Elastic Solids] (1973).

[20] Nadella, K. S. and Cesnik, C. E. S., "Local interaction simulation approach for modeling wave propagation in composite structures," CEAS Aeronautical Journal 4, 35-48 (2013).

[21] Murphy, K., [Machine Learning: A Probabilistic Perspective], The MIT Press (2012).

[22] Zellner, A., "On assessing prior distributions and Bayesian regression analysis with g prior distributions," in [Bayesian Inference and Decision Techniques, Studies of Bayesian and Econometrics and Statistics], Goel, P. and Zellner, A., eds., 233-243, Elsevier Science Ltd. (1986). 